\title{
An AST/RO Survey of the Coalsack
}

\author{
Wilfred Walsh and Kecheng Xiao \\ Harvard-Smithsonian Center for Astrophysics, 60 Garden St, MS12, \\ Cambridge MA 02138, USA
}

\begin{abstract}
Selected regions of the southern molecular cloud complex, the Coalsack are being imaged at sub-millimeter wavelengths using the Antarctic Sub-millimeter Telescope and Remote Observatory (AST/RO) located at the South Pole.
\end{abstract}

The Coalsack is one of the most prominent naked-eye features of the Southern sky, covering about 30 square degrees adjacent to the Southern Cross with 0.7-0.24 mag of extinction. Its proximity (about 180pc) make it an ideal candidate for studying the structure, composition and dynamics a large molecular cloud. Unlike other prominent dark clouds, the Coalsack has generally been thought to show no evidence of collapse leading to star formation. We have selected regions of the Coalsack to image at submillimeter wavelengths: this work is currently in progress. The AST/RO observations consist of a fully-sampled survey in the $230 \mathrm{GHz} \mathrm{CO}(2-1)$ line over the entire extent of the Coalsack, together with smaller regions imaged in other $\mathrm{CO}$ isotopomers and the $461 \mathrm{GHz}$ $\mathrm{CO}(4-3)$ and $492 \mathrm{GHz}$ CI lines. We have already detected extended $\mathrm{CO}(4-3)$ emission at several locations. A preliminary LVG model for one position suggests that localized warm $(25 \mathrm{~K})$ gas does exist in the Coalsack and that collapse leading to star formation, if not already occurring, may be imminent.
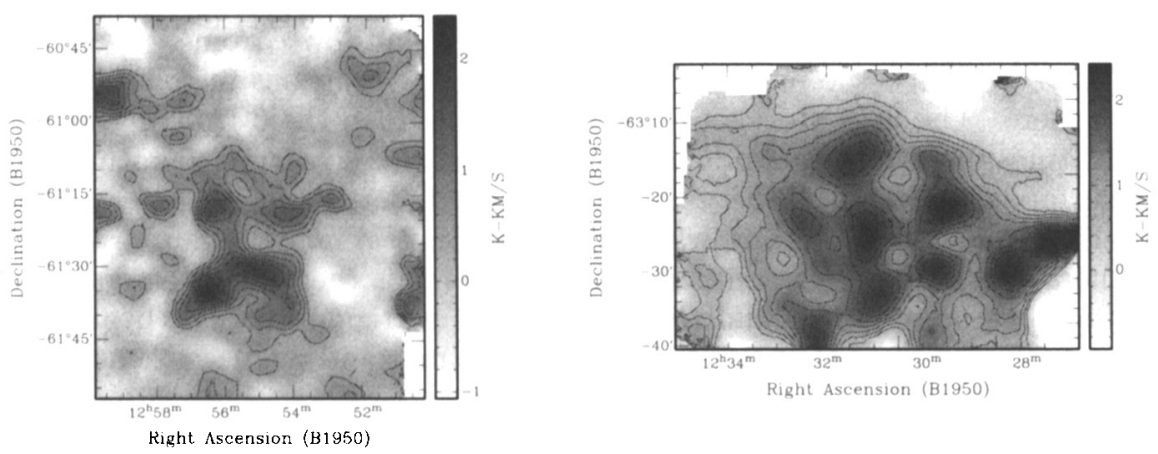

Figure 1. Two regions of the Coalsack imaged in the $\mathrm{CO}(4-3)$ line, shown with contours from 0.5 to $2.0 \mathrm{~K} \mathrm{~km} / \mathrm{s}$ in $0.25 \mathrm{~K} \mathrm{~km} / \mathrm{s}$ steps. 